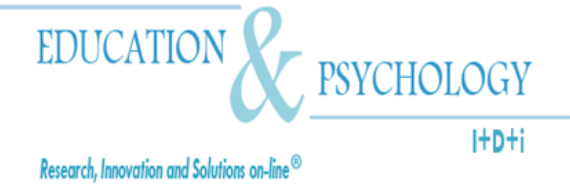

\title{
La psicopedagogía como ámbito científico-profesional
}

\section{Lidia Cabrera Pérez ${ }^{1}$, José Tomás Bethencourt Benítez ${ }^{2}$}

\author{
${ }^{1}$ Área de Métodos de Investigación y Diagnóstico en Educación, \\ Universidad de La Laguna \\ ${ }^{2}$ Departamento de Psicología Evolutiva y de la Educación, \\ Universidad de La Laguna
}

\section{España}

Correspondencia: Lidia Cabrera Pérez. Facultad de Educación. Campus Central. Universidad de La Laguna. 38204 La Laguna. Santa Cruz de Tenerife. España. E-mail: dcabrera@ull.es

(C) Education \& Psychology I+D+i and Editorial EOS (Spain) 


\section{Resumen}

En este trabajo se hace un análisis de la psicopedagogía como ámbito científico y profesional desde su origen epistemológico hasta su configuración actual como espacio profesional autónomo. En su consolidación en el Estado Español se resalta el papel preponderante desempeñado por las universidades con su actividad investigativa y formativa, la reivindicación social de los movimientos de renovación pedagógica y el desempeño profesional de los equipos psicopedagógicos en los centros escolares. Para ilustrar el desarrollo tanto científico como profesional de esta disciplina presentamos los resultados de dos estudios: (a) un estudio de revisión bibliométrica sobre las publicaciones psicopedagógicas en lengua castellana de los últimos diez años, y (b) un estudio de seguimiento de los egresados de Psicopedagogía en la Universidad de La Laguna. Finalmente, se valoran y pronostican las implicaciones que la consolidación de la psicopedagogía como disciplina tendrán en el diseño de los próximos títulos específicos de grado y postgrado universitarios.

Palabras Clave: disciplina psicopedagogía; origen conocimiento psicopedagógico; inserción laboral titulados en psicopedagogía; producción científica psicopedagogía.

Recibido: 22/12/09 Aceptación Inicial: 24/12/09 Aceptación Definitiva: 29/07/10 


\title{
School psychology as a scientific and professional sphere
}

\begin{abstract}
In this paper an analysis of the psychopedagogy is done as scientific and professional discipline from its origin epistemology up to the current configuration as professional autonomous space. In its consolidation in the Spanish State there is highlighted the preponderant role played by the universities in the research and training activity, the social recovery of the movements of pedagogic renovation and the professional performance of the psychopedagogues. We are showing the results of two studies to illustrate the development so much scientific as professional of this discipline: (a) A review study of the psychopedagogy publications in spanish language of last ten years, and (b) A study about the undergraduates of La Laguna University. Finally, there are valued and predict the implications that the consolidation of the psychopedagogy will have in the degrees and postdegrees university.
\end{abstract}

Keywords: Disciplines psychopedagogy; Origin knowledge of psychopedagogy; employment of psychopedagogy; Scientific production of psychopedagogy.

Received: 12/22/09 Initial Acceptance: 12/24/09 Definitive Acceptance: 07/29/10 


\section{Introducción}

La preocupación por la educación y el estudio del comportamiento humano ha estado presente en toda la historia de la humanidad. Sin embargo, el nacimiento de las ciencias pedagógicas y psicológicas como disciplinas encargadas de construir conocimiento científicoexperimental es muy reciente. Apenas cuentan, una y otra, con poco más de un siglo de historia. Este conocimiento se inicia, consolida y sistematiza en el entorno de la comunidad científica tras el inicio y aplicación de la experimentación y el método científico a los problemas de una y otra disciplina, a finales del siglo XIX. Otra cuestión es si obviamos o no el conocimiento anterior, no sólo porque constituye el cimiento del conocimiento actual, sino por la emergencia posterior de otros métodos alternativos al experimental que, actualmente, le dotan del mismo rigor. En ese momento, sin embargo, el discurso positivista de la pedagogía y la psicología científica, supuso un enorme despliegue de acciones y modelos teóricos en toda Europa y América.

La psicopedagogía es una consecuencia directa del nacimiento de la pedagogía y la psicología experimental, sobre todo aplicada al ámbito de las deficiencias físicas y mentales. La preocupación que existía en ese momento por la infancia (paidología y child study) junto a la obligatoriedad de la enseñanza, y la emergencia de la experimentación aplicada al estudio de las diferencias individuales, sientan las bases científicas disciplinares y sociales del profesional actual de la psicopedagogía. Había que educar a la ciudadanía, pero para ello había que construir el conocimiento que permitiera la explicación de su comportamiento individual, ante situaciones específicas de aprendizaje. En un contexto fuertemente influenciado por las ideas de Darwin y la selección natural de las especies, cobran gran relevancia la Pedagogía Experimental y Diferencial. De forma paralela, la predominante preocupación por la infancia estrecha lazos entre profesionales de la medicina y de la educación para dar respuesta a la educación especial, higiene escolar y la infancia desamparada (pedagogía médica).

Actualmente, estas disciplinas han sido reemplazadas por otras aglutinadas en torno a la psicopedagogía, tales como: atención a las necesidades educativas específicas; orientación educativa, vocacional y profesional; evaluación psicopedagógica y diagnóstico de necesidades educativas; estrategias y estilos de enseñanza y aprendizaje, intervención psicopedagógica en áreas de apoyo al currículo educativo, etc. Objetos de estudio clásicos de la pedagogía dife- 
rencial fueron $^{1}$ : (1) En relación a las edades (preescolar, primaria, media, universitaria, edad adulta y vejez); (2) En relación a las unidades sociales (educación familiar, institucional y ambiental); (3) En relación a las deficiencias (físicas, psíquicas, sociales, intelectuales, interculturales, del lenguaje, etc.); etc.

La psicopedagogía por tanto, como disciplina científico-profesional, cuenta con antecedentes históricos bien delimitados en diferentes países del continente europeo y americano. Como muestra de ello puede citarse el proceso de desarrollo e implantación tanto científica como profesional de la psicopedagogía realizado en Francia. Para Andrey y Le Men (1970) la obra del autor francés Henri Wallon (1879-1962) representa la mayor concreción de la integración de la psicología en la escuela, y una consecuencia de dicha obra es la fundación en el año 1925 del primer Laboratorio de Psicopedagogía en el seno de la escuela pública, que dio paso en el año 1927 a la creación del Laboratorio de Psicología del Niño.

En 1946 Wallon creó un centro psicopedagógico modélico para otros muchos, de tal manera que, en 1970 se creó la Asociación Francesa de Centros Psicopedagógicos que integraba a unos 20 centros de esa naturaleza. Las decisivas y extensas contribuciones del psicopedagogo francés Wallon han quedado reflejadas en los trabajos de Andrey y Le Men (1970) ya citado, en el monográfico $n^{\circ} 6$ coordinado por el psicólogo Jesús Palacios en la revista Cuadernos de Pedagogía del año 1980, en los artículos de la revista Infancia y Aprendizaje correspondientes a los números 3 y 4 (año 1978) y n 8 (año 1979) y finalmente en la obra de su propio discípulo René Zazzo del año 1975 y de título Psicología y Marxismo.

Fue, precisamente, Zazzo, quien sucedió a Wallon en el Laboratorio de Psicología del Niño y publicara otras obras como: El devenir de la inteligencia (1946), Nueva escala métrica de inteligencia (1966), Los niños de 6 a 12 años (1969), Los débiles mentales (1969) y Manual para el examen psicológico del niño (1970).

En España, la institucionalización de estos estudios se produce de forma más tardía que en el resto de Europa. Un ejemplo de ello es que mientras en Francia, país vecino del Estado Español, se producía una importante reforma de la enseñanza en el año 1947, la famosa

\footnotetext{
${ }^{1}$ Algunas publicaciones de referencia fueron: Fernández-Sarramona, (1977). Aspectos diferenciales de la educación. Barcelona: CEAC. García Hoz, V. (1987). Principios de la pedagogía sistemática. Madrid: Rialp.
} 
reforma conocida con el nombre de sus promotores Langevin-Wallon, en la cual se fijaron las principales funciones del psicopedagogo, en España se produce 25 años mas tarde, en los años 70 con la Ley General de Educación, tomando como referencia la francesa. En España el conocimiento relativo a la psicología y pedagogía se incluía en la licenciatura de filosofía. En 1902 se crea la primera cátedra de Psicología Experimental y en 1904 la de Pedagogía Experimental, ambas en La Universidad de Madrid. Mientras, en el resto de Europa y en Estados Unidos ya se institucionalizaba la psicopedagogía como disciplina encargada de atender las necesidades especiales de los estudiantes.

El panorama educativo español, sin embargo, se nutría de ese conocimiento que venía de fuera, sobre todo de la mano del profesorado de las Escuelas Normales, y los inspectores de enseñanza primaria, quienes se encargaron de introducirlo y difundirlo a través de publicaciones como: el Boletín de la Institución Libre de Enseñanza, la Revista de Pedagogía, o la Revista de las Escuelas Normales. Estudios recientes sobre las aportaciones españolas al panorama mundial de la psicopedagogía ${ }^{2}$, como las de la Institución Libre de Enseñanza, profesionales de la pedagogía, de la medicina como Ramón y Cajal, juristas, de la sociología, y más tarde de la psicología, ponen de manifiesto un discurso psicopedagógico en España, que se plasmó en la formación de profesionales (como el curso de técnicas psicopedagógicas organizado por el Museo Pedagógico Nacional) e instituciones educativas que llevan el nombre de psicopedagógico, tal como sucedía en el escenario europeo ${ }^{3}$. En el entorno de los años 20 y 30 el término psicopedagógico se usaba frecuentemente para denominar las prácticas educativas especiales, la orientación profesional que empezaba a gestarse, las orientaciones en la reeducación de menores, etc. La revista Infancia Nostra, fundada en 1922 en Barcelona, contaba con una sección de psicopedagogía, donde distintos profesionales comunicaban sus experiencias en torno a la reeducación de menores.

Toda esta construcción y sistematización del entorno psicopedagógico que se produce en la primera mitad del siglo XX en España se ve truncada por la dictadura franquista. Se eclipsan las ideas educativas de atención personalizada y especializada, y las personas propulsoras de estos modelos y métodos, así como grandes teóricos e investigadores se van al exilio.

\footnotetext{
2 Estudios sobre la Historia de la Psicopedagogía en España (Carrión, 1996; Cerezo, 1996, 2001; Bisquerra, 1996; Moreu, 2002; Moreu y Bisquerra, 2002).

3 A modo de ejemplo, en 1914, la pedagoga Francisca Rovira, propone un "método psicopedagógico" para el tratamiento de la sordera, en Barcelona. Ese mismo año, Anselmo González, profesor de la Escuela de Estudios 
Se inicia un periodo de incertidumbre y desorden epistemológico para ciertos ámbitos del conocimiento psicopedagógico, que más tarde buscan un lugar en el entorno de las denominadas Ciencias de la Educación. A pesar de este periodo de latencia de la psicopedagogía en España, en casi todas las obras de pedagogía diferencial y educación especial se observa una constante referencia a la psicopedagogía, y en la reeducación de menores siempre se habla de métodos psicopedagógicos. El término psicopedagogía ha aparecido siempre, para hacer referencia al conocimiento psicopedagógico, en todos los diccionarios y enciclopedias pedagógicas, como en el diccionario de psicopedagogía, diccionario de las ciencias de la educación, enciclopedia práctica de pedagogía, enciclopedia temática de educación especial, diccionario enciclopédico de educación especial, etc.

Sin embargo, el conocimiento psicopedagógico se fragmenta, por lo que los distintos ámbitos se van desarrollando al amparo de la pedagogía y la psicología de manera independiente, buscando formas específicas y diferenciadas en unos casos, pero solapándose en otros. El carácter bicefálico de la psicopedagogía viene dado, por tanto, de la confluencia y solapamiento de dos disciplinas y dos profesionales que se han ido desarrollando de forma autónoma, como ha quedado manifiesto en innumerables publicaciones (Coll, 1996; Gonzalo, 1991; Ortiz, 2000; Poveda, 2003; Rigo, 1990).

La fragmentación histórica en el corpus de conocimiento y la aglutinación actual del profesional de la psicopedagogía es la que ha provocado las fuertes tensiones, disputas y enfrentamientos gremiales entre profesionales de la psicología y la pedagogía, las cuales no tienen sentido desde las actuales perspectivas teóricas del propio conocimiento psicopedagógico, más ambientalistas, más constructivistas y basadas en enfoques más de desarrollo que terapéuticos, y en dinámicas más colaborativas. La ampliación de funciones de la intervención, así como de los ámbitos de acción, han creado una intersección difícil de diferenciar. En este trabajo nos proponemos contribuir a superar esas limitaciones defendiendo la posición de la psicopedagogía como ámbito interdisciplinar, en la cual tienen que confluir o converger necesariamente la psicología escolar y de la educación, la orientación educativa, la educación especial, el diagnóstico o evaluación psicopedagógica, y las bases didácticas para la intervención, entre otra. A la vez que defendemos la formación disciplinar y profesional básica, y no la especialización en uno u otro ámbito (psicológico y pedagógico). copedagógico para referirse a este conocimiento (Moreu y Bisquerra, 2002). 
Las contribuciones específicas de la psicología a la psicopedagogía en el Estado Español, tanto en el terreno científico como profesional, pueden ser revisadas en la obra de Báez y Bethencourt (1992). Estos autores concluyen que la actividad investigativa y formativa de las universidades, la reivindicación social de los movimientos de renovación pedagógica y el desempeño profesional de los psicólogos y las psicólogas en los centros escolares privados, contribuyó a que las instancias gubernamentales y administrativas fueran aceptando la lenta, tardía, progresiva y precaria implantación e institucionalización de los servicios psicopedagógicos en el sistema público de educación.

\section{Reforma social e institucionalización de la psicopedagogía en el Estado Español}

Antes de definir o conceptualizar el campo disciplinar de la psicopedagogía como nosotros la entendemos en el momento actual, haremos un breve resumen de la ordenación administrativa que ha determinado la aglutinación actual del conocimiento psicopedagógico.

Como ya hemos adelantado, las reformas educativas que se fueron produciendo en Europa a mitad del siglo pasado, en el caso de España se concretan con la promulgación de la Ley General de Educación (LGE) de 1970, en la cual se reconocía el derecho del alumnado a recibir servicios de orientación escolar, personal y profesional. Pero tal derecho se ve implementado muchos años después, cuando en 1977 se dicta la orden ministerial por la que se crearon los Servicios Provinciales de Orientación Escolar y Vocacional (SPOEV), y más tarde los equipos multiprofesionales (EM), los primeros en el ámbito de la orientación y los segundos en el de la educación especial. En el marco social, sin embargo, sigue imperando el término natural de psicopedagógico, y dos años más tarde, con motivo de la instauración de los ayuntamientos democráticos, se crean los Servicios Psicopedagógicos Municipales (SPM), con la finalidad de atender áreas educativas no cubiertas por la escuela.

La transferencia de competencias en educación a las primeras Comunidades Autónomas en 1982, inicia una diferenciación en la organización de la acción psicopedagógica en el escenario educativo del Estado Español. En 1983 en Cataluña empiezan a funcionar los Equipos de Asesoramiento y Orientación Psicopedagógica. En la Comunidad valenciana los Servicios Psicopedagógicos Escolares (1984). En Galicia, los Equipos Psicopedagógicos de Apoyo a la Escuela (1985), y así sucesivamente. También hay 
que indicar que en otras comunidades autónomas no se introduce todavía el término psicopedagógico, como en el País Vasco (Centros de Orientación Pedagógica: COPs), Navarra (Servicios de Ordenación Académica e Innovación Educativa); Andalucía (Equipos de Promoción y Orientación Educativa: EPOEs.); etc.

La reforma educativa que se inicia en España en los años 80, culmina con la promulgación en 1990 de la Ley Orgánica de Ordenación General del Sistema Educativo (LOGSE), y la aglutinación del conocimiento psicopedagógico que había estado diseminado y fragmentado. Desde la LOGSE se impulsa la orientación e intervención psicopedagógica en todos los centros de enseñanza obligatoria, con el fin de que den apoyo y asistencia a las necesidades de los centros, el profesorado, el alumnado y sus familias. Esta asistencia constituye uno de los capítulos de excelencia y calidad educativa del nuevo sistema que pretende la Ley. La LOGSE demanda al sistema educativo: un sistema de apoyo al currículo de orientación educativa e intervención psicopedagógica, una mayor calidad de la enseñanza, y una personalización de los aprendizajes. Para ello se institucionaliza dentro del sistema educativo la figura profesional del psicopedagogo/a, que hasta ese momento había estado diversificada, o repartida entre dos profesionales (pedagogo/a y psicólogo/a escolar).

La ordenación del panorama concluye con la fusión de los tres servicios hasta el momento existentes: Servicios de Orientación Escolar y Vocacional (SOEV), los Equipos Multiprofesionales (EM), y los Servicios Psicopedagógicos Municipales (SPM). La propuesta del Ministerio (MEC, 1990) es un modelo global y único de orientación e intervención psicopedagógica, a través del funcionamiento de departamentos de orientación en centros de enseñanza secundaria (especialidad de pedagogía y psicología), equipos sectoriales que ofrezcan apoyo a los centros de infantil y primaria, y equipos específicos para la atención del alumnado con necesidades educativas especiales. La mayoría de las Comunidades Autónomas secundan esa propuesta. Además de las funciones de evaluación y orientación al alumnado, el Ministerio de Educación hace una relación de ámbitos necesitados de aportación técnica de carácter psicopedagógico. Destacamos el desarrollo de actitudes, normas y valores; las estrategias de enseñanza y aprendizaje; cómo enseñar y aprender en materias específicas o ante dificultades concretas; cómo desarrollar técnicas de estudio y aprendizaje; estrategias de solución de problemas y procesamiento de la información; mecanismos para aprender a pensar y aprender a aprender; etc. (MEC, 1990). En este documento se resalta el derecho del alumnado a recibir atención y orientación individualizada a través de un conjunto de servicios y actividades pro- 
pias de la psicopedagogía: tutoría académica, departamentos de orientación y equipos interdisciplinares de zona o sector.

En 1991, el MEC regula el ejercicio profesional de la psicopedagogía en centros educativos para su propio territorio, y dictamina que las tareas de intervención psicopedagógica sean llevadas a cabo por los Equipos de Orientación Educativa y Psicopedagógica: EOEPs. (Orden de 14/6/91 y Orden de 9/12/92 por la que se regula la estructura y funciones de los EOEPS), y los Departamentos de Orientación de los Institutos de Educación Secundaria (Real Decreto 199/1993 y R.D. 7/5/96 por el que se aprueba el Reglamento Orgánico de los Institutos de Educación Secundaria). En la Comunidad Autónoma Canaria esta reforma se produce en 1995, con la constitución de los Equipos de Orientación Educativa y Psicopedagógicos: EOEPs. (Decreto 23/95 -BOC n ${ }^{\circ} 34$, de 20/3/95- por el que se regula la orientación educativa la Comunidad Autónoma Canaria).

El acceso laboral a los distintos espacios profesionales ha sido posible tanto desde la psicología como desde la pedagogía, exigiendo a los pedagogos y pedagogas retomar la formación psicológica recibida en su formación inicial, y a los/as psicólogos/as "subsanar su carencia de conocimientos sobre los procesos educativos a los que no tuvieron acceso en el transcurso de su formación inicial" (Coll, 1996:43). Se trataba pues de fomentar la formación de profesionales competentes en todas las acciones psicopedagógicas que el sistema educativo y social demanda, independientemente de las divisiones disciplinares.

Pero el Ministerio de Educación y Ciencia, con la intención de "compensar las lagunas detectadas en la formación inicial, tanto de psicólogos escolares como de pedagogos especializados en orientación, para la realización de actividades y tareas típicamente psicopedagógicas" (Coll, 1996, p. 43), institucionaliza los Estudios Oficiales de Psicopedagogía, que son regulados a través del Real Decreto 916/1992 de 17 de Julio, con el apoyo de los profesionales de uno y otro ámbito. Gonzalo (1991) es muy explícito al respecto, cuando dice: "es evidente que no cualquier psicólogo o pedagogo por el mero hecho de tener la titulación, posee ya el perfil apropiado para las funciones de orientación e intervención psicopedagógica". Continúa este autor diciendo que "acerca de los rasgos de profesionalidad y especialización que se derivan del texto del libro Blanco y del Documento sobre Orientación Educativa e Intervención Psicopedagógica, éstos parecen reclamar un perfil definido por conocimientos teóricos y competencias prácticas que capacite en todas aquellas intervenciones en el campo 
psicopedagógico a nivel de institución, en aspectos referidos a procesos de enseñanza aprendizaje, o al conocimiento del alumnado en toda su diversidad" (pág. 202).

Este proyecto amplió el debate epistemológico entre pedagogos y psicólogos de todo el Estado. Pero la consolidación y ampliación del espacio de intervención psicopedagógica dentro del sistema educativo, hacían evidentes las necesidades de la confluencia, tal como quedaban recogidas en las palabras de uno de los promotores de tales estudios universitarios de psicopedagogía, "las disfunciones y problemas derivados del esfuerzo por mantener hasta sus últimas consecuencias el carácter estanco e impermeable de ambas tradiciones cuando confluyen en un mismo espacio profesional" (Coll, 1996, p. 42). Un ejemplo de ello es la imposibilidad de separar en la práctica funciones de unos y otros, pues ambos "acaban asumiendo en realidad las mismas funciones y realizando actividades y tareas muy parecidas" (Coll, 1996, p. 42).

Con la adaptación al espacio europeo de educación superior, se abre de nuevo el debate, pero esta vez en torno a la modalidad de formación del psicopedagogo, que están aprovechando una vez más los gremios profesionales para reivindicar su espacio diferenciado del conjunto. ¿Constituye el conocimiento psicopedagógico un corpus disciplinar básico profesionalizador, o una formación especializada dentro de los estudios de pedagogía y psicología respectivamente? Con esta indefinición entramos no sólo en el debate sobre una formación de grado o postgrado, sino en qué ámbito y dónde se organiza esa formación especializada: ¿psicología escolar?, ¿orientación educativa?, ¿educación especial?... ¿Cuántos masters tendrá que hacer entonces el profesional de la psicopedagogía para abordar todas sus funciones profesionales? De forma simultánea a este debate, se inicia la reforma del título de postgrado de "Profesor de enseñanza secundaria obligatoria y bachillerato, formación profesional y enseñanzas de idiomas" que incluye entre sus especialidades la de "orientación educativa", con la finalidad de formar al profesorado que ocupará las plazas de los departamentos de orientación en secundaria (Resolución de 17 de diciembre de 2007, BOE nº. 305).

Está aún por determinar el acceso a dicha especialidad, en principio propia para licenciados en psicopedagogía, únicos titulados en la actualidad con formación básica en orientación. Si desaparecen los estudios de grado de psicopedagogía ¿hemos de entender que el acceso vuelve a ser para los pedagogos y psicólogos? Por otro lado, aparte de esta necesidad formativa básica para el acceso a la especialidad de "formación del profesorado", se requiere de 
la formación básica específica para otros ámbitos profesionales de considerable arraigo social en la actualidad. Por citar algunos de ellos tenemos entre los más considerables: dentro del sistema educativo, la intervención psicopedagógica en infantil y primaria; y fuera de él, la orientación profesional y laboral; intervención psicopedagógica comunitaria (gabinetes psicopedagógicos, servicios de educación especial, estimulación cognitiva con mayores, etc.). Algunos de estos ámbitos que han ido emergiendo fuera de la escuela exigen de un conocimiento psicopedagógico más allá del tradicional desarrollado por la psicología educativa, la orientación educativa y la educación especial, por citar las tres grandes áreas con mayor definición. Por lo que estamos más cerca que nunca de una disciplina científica y profesional aglutinadora e independiente de otros perfiles formativos como los del pedagogo o el psicólogo. En el siguiente apartado nos acercamos a este corpus de conocimiento desde la producción y el conocimiento científico.

\section{Conceptuación disciplinar y espacio profesional}

La evidencia muestra: primero, que la psicopedagogía tanto como disciplina científica como de formación para el ejercicio profesional goza de carácter autónomo desde hace más de un siglo; segundo, que la psicopedagogía, o términos afines, surge para definir un tipo de conocimiento pedagógico y psicológico, el referido a situaciones educativas con necesidades especiales, o diferenciadas; tercero, los psicopedagogos y psicopedagogas, por tanto, desarrollan su ejercicio profesional en contextos educativos formales y no formales, y han sido siempre profesionales de la intervención psicopedagógica. El conjunto de conocimiento teórico y aplicado que se ha derivado en los últimos 17 años en España lo evidencia. En la tabla 1 mostramos una síntesis de un estudio de revisión de los artículos indexados en la base de datos DIALNET, y que incluyen en el título los términos "psicopedagógico", "psicopedagógica”, o "psicopedagogía". El conjunto lo constituyen 943 referencias, que hemos organizado atendiendo a las siguientes categorías de análisis: funciones profesionales; conceptualización, formación y profesionalización; teorías, modelos y métodos de intervención; y finalmente, áreas de intervención. 
Tabla 1. Resultados estudio de revisión sobre publicaciones psicopedagógicas

Funciones profesionales

Asesoramiento (centros, profesorado y ámbitos de intervención, etapas educativas), adaptaciones curriculares

Coordinación otros servicios comunitarios: relaciones interprofesionales.

Dinamización de la intervención (nueva funciones educativas) y mediación

Diagnóstico y evaluación psicopedagógica, detección y análisis de necesidades

Metodologías de Investigación psicopedagógica y estrategias de investigación aplicadas

Experiencias de innovación en psicopedagogía

Conceptuación y profesionalización

Conceptuación de la psicopedagogía: perfil profesional, formación (inicial y continua) y desarrollo profesio-

nal (competencias)

Formación en los estudios de psicopedagogía (practicum)

Historia, concepto y modelos de orientación e intervención, enfoques teóricos de intervención: cómo, dónde, con quién, etc.

Asociaciones profesionales

Ética y deontología profesional

Intervención psicopedagógica y calidad

Modelos de equipos psicopedagógicos: centros escolares, otras instituciones educativas, comunidades y legislación

Gabinetes psicopedagógicos universitarios

Intervención hospitalaria

Intervención psicopedagógica con mayores

Intervención psicopedagógica comunitaria y compensatoria (entornos desfavorecidos, actitudes hacia la lectu-

ra, enseñanzas disciplinares -danza, idiomas, valores para el consumo, integración de minorías, etc.)

Orientación vocacional y sociolaboral

Investigación psicopedagógica en educación social (adaptación social), educación no formal

Niños en contextos familiares en riesgo o problemáticos (maltrato, abuso sexual), y familias de niños con dificultades

Participación de padres en la escuela y contexto familiar

Teorías, modelos y métodos de intervención

Modelos y teorías psicológicas aplicadas

Métodos y estrategias de intervención psicopedagógica: individualizada, retraso y dificultades académicas

Programas de intervención

Medios educativos y materiales didácticos (hardware y software para la diversidad)

Modelo de enseñanza tele-enseñanza, nuevas tecnologías, televisión, recursos electrónicos, entornos virtuales 13

\section{Áreas de intervención}

Profesorado: formación psicopedagógica, pensamiento, actitudes y calidad de la enseñanza

Estrategias y dificultades de aprendizaje (matemáticas, lengua, lectura, comprensión) enseñanza estratégica, enfoques de aprendizaje, reeducación cognitiva, etc.

iscapacidad, diversidad y NEE, trastornos del desarrollo, altas capacidades, inclusión

Desarrollo del lenguaje y la comunicación

Educación temprana e infantil

Intervención en educación secundaria

Enseñanza e intervención específica: valores, convivencia, desarrollo moral, autoconcepto, motivación, crea-

tividad, educación emocional e interpersonal, fobia escolar, etc.

Psicomotricidad

Aptitudes y rendimiento

Hiperactividad

Contexto escolar y ambientes de aprendizaje

Acción tutorial

Riesgo social en la escuela

Otras áreas de apoyo u objeto de intervención (educación física, publicidad y alcohol, comportamiento cívico y social, exploración y creatividad) 
Esta pequeña representación del conjunto del conocimiento científico psicopedagógico evidencia: la definición de un corpus propio de conocimiento, la emergencia constante de nuevos ámbitos de acción profesional, dentro y fuera de la escuela, y la consecuente necesidad de una formación disciplinar específica, con la suficiente amplitud para abordar las competencias profesionales generales y específicas del profesional en psicopedagogía, así como de especialización en ámbitos específicos de intervención y estrategias de investigación.

Otra muestra de la consolidación de la disciplina es la celebración de congresos estatales e internacionales, la edición de revistas con título psicopedagógico, las asociaciones profesionales y las instituciones y servicios sociales con tal denominación.

a) Congresos y eventos científicos. A pesar de que el conocimiento psicopedagógico tiene amplios espacios en congresos específicos, como es el de Modelos de Investigación en Educación; las Jornadas de Educación Especial; o los Congresos de Pedagogía, Psicología y Educación; etc., Destaca la celebración sistemática de congresos propiamente psicopedagógicos como son las Jornadas Nacionales de la Asociación Española de Orientación y Psicopedagogía, celebradas anualmente; las Jornadas de Psicopedagogía de la Universidad de Deusto, celebradas en Bilbao (2002); o el Congreso Internacional de Psicopedagogía celebrado en Melilla (2006), como ejemplo de congresos específicos de psicopedagogía; etc.

b) Edición de revistas. Revistas especializadas en temas psicopedagógicos: “Ámbitos de Psicopedagogía; Revista Catalana de Psicopedagogía y Educación"; "Revista Española de Orientación y Psicopedagogía"; "EduPsykhé: Revista de Psicología y Psicopedagogía"; "Revista Galega de Psicopedagoxia"; "Revista de Orientación Psicopedagógica"; "Revista Electrónica de Investigación Psicoeducativa y Psicopedagógica, Electronic Journal of Research in Educational Psychology"; etc.

c) Asociaciones profesionales. A pesar de que no existe todavía un colegio oficial de psicopedagogos, los colectivos profesionales con estas finalidades han pasado a denominarse psicopedagógicos. El ejemplo más evidente es la Asociación Española de Orientación y Psicopedagogía (AEOP), la cual en el año 2004, se incorpora a la "Confederación de Organizaciones de Psicopedagogía y Orientación de España" (COPOE), que se crea en ese mismo año, con la finalidad de aglutinar a colectivos de profesionales que persiguen objetivos y competencias similares. La COPOE, integra en estos momentos un considerable número de asociaciones psicopedagógicas como: Asociación Aragonesa de Psicopedagogía; Asociación Canaria de Orientación y Psicopedagogía; Asociación Madrileña de Orientación y Psicopeda- 
gogía; Asociación de Psicopedagogía de Euskadi; Asociación Psicopedagógica de la Universidad Oberta de Catalunya; etc.

d) Instituciones y servicios sociales. El ejercicio profesional fuera del sistema educativo ha dejado de denominarse pedagogo especializado en educación especial, orientación, o psicólogo escolar, y prevalecen centros y servicios psicopedagógicos. Los gabinetes privados adquieren denominaciones diversas, en función de sus finalidades, pero casi siempre aludiendo a la acción o intervención psicopedagógica. Algunos ejemplos son: "gabinete de psicopedagogía"; "centro de evaluación e intervención psicopedagógica" "servicio de intervención psicopedagógica"; "gabinete de psicopedagogía y logopedia", etc. Las instituciones públicas con finalidades educativas van incorporando servicios con esta denominación, como los asesores y servicios psicopedagógicos universitarios, hospitalarios, municipales, etc. Finalmente, en contextos objeto de intervención grupal o comunitaria, los programas y planes diseñados son denominados de intervención psicopedagógica, etc.

En el plano internacional, Moreu y Bisquerra (2002), hacen un estudio epistemológico del término psicopedagógico, comparando distintos escenarios y tradiciones (anglosajona, francófona, alemana, italiana, etc. $)^{4}$, y concluyen que "la psicología de la educación en estas áreas ha de considerarse, desde el punto de vista epistemológico, como una ciencia basal de la psicopedagogía" (p. 22), aportando algunos fundamentos del comportamiento.

Parte de esta realidad también se constata en la existencia y la actividad de diversas asociaciones profesionales especializadas en ámbitos parciales de la psicopedagogía, como son la ISPA (Asociación Internacional de Psicología Escolar), la AIOSP (Asociación Internacional de Orientación Educativa y Profesional), o las AERA y EERA (Asociaciones Americana y Europea respectivamente de Investigación en Educación). Su justificación radica en modelos alternativos de ordenación profesional. En España, Latinoamérica y otros países de la Unión Europea, como Bélgica o Alemania, se centraliza en el psicopedagogo las funciones indirectas de tres profesionales: el orientador escolar, el maestro especialista en educación especial y psicopedagogía, y el psicólogo escolar. Por otro lado, los modelos actuales de intervención psicopedagógica en España superan los modelos de intervención directa y clínicos

\footnotetext{
${ }^{4}$ Las primeras referencias del término "psicopedagogía" como tal aparecen en el ámbito francófono (Francia, Bélgica, y la Escuela de Ginebra en Suiza), en 1908, para referirse a las técnicas psicométricas destinadas a la clasificación de los escolares. En Italia también se hizo un uso temprano del término (1912), para denominar a profesionales que debían hacer una evaluación de la deficiencia mental, que completara el examen médico (Moreu y Bisquerra, 2002).
} 
anteriores, para centrarse en modelos de intervención colaborativa, por lo que las funciones del orientador son más indirectas y de asesoramiento, lo que justifica la centralización en un único profesional.

\section{Desarrollo profesional actual y perspectivas futuras de la psicopedagogía}

En la sociedad actual se identifican cada vez nuevas necesidades educativas que requieren intervención o acción psicopedagógica. Muestras de ello son el incremento de publicaciones en ámbitos específicos de intervención (ver tabla 1), y la inserción laboral de los licenciados en psicopedagogía. En un estudio realizado por Álvarez, Bethencourt y Cabrera (2000), sobre la transición al mercado laboral de las dos primeras promociones de egresados en psicopedagogía en la Universidad de La Laguna, mostraron que en los servicios de orientación psicopedagógica de los centros educativos solo se había colocado un $8 \%$ de los egresados.

En otro estudio similar realizado por Cabrera y Bethencourt, durante el año 2009 y aún sin publicar, encontramos que los psicopedagogos y psicopedagogas en las Islas Canarias están trabajando: en los servicios de orientación psicopedagógica de los centros educativos (12\%); de orientadores laborales (fuera del entorno escolar) (7\%); de maestros de infantil y primaria (28\%); en centros de educación y desarrollo infantil (5\%); en servicios sociales de ayuntamientos $(6 \%)$; en programas comunitarios de intervención psicopedagógica $(29 \%)$; en centros de psicomotricidad (2\%); en drogodependencias (1\%); educación de adultos (3\%); en mediación intercultural (1\%); en intervención familiar (1\%); en centros de menores $(2 \%)$; en centros de educación especial y atención a la discapacidad (3\%).

Otras evidencias son los resultados de estudios sobre el desarrollo del practicum de la titulación y de egresados en psicopedagogía, que muestran un desarrollo profesional bastante diverso (Cano, 2003; Castro, Rodríguez y Cubo, 2001; Cubo y Montenero, 2000; Hidalgo e Hidalgo, 2003; López, 2001; Ocampo y Cid, 2001; Pérez y Torres, 1998; Tello, 2007).

Los datos son más que esclarecedores para concluir que el ejercicio profesional del psicopedagogo trasciende más allá de los estrictos servicios psicopedagógicos escolares y servicios de orientación, y que la acción psicopedagógica también se extiende más allá de los 
escolares con necesidades educativas especiales y con necesidades de atención u orientación específica.

Los ámbitos de acción profesional objeto de la intervención psicopedagógica que se han ampliado en los últimos años han sido:

1. En el entorno escolar: tareas y actividades de coordinación y asesoramiento de profesorado y servicios escolares, y de éstos con otros servicios comunitarios; mediación y gestión de conflictos interpersonales e interculturales; compensación de diferencias educativas de origen cultural y social; gestión y fomento de la participación de las familias en el desarrollo educativo de sus hijos e hijas; asesoramiento al profesorado en tareas de acción tutorial; asesoramiento y coordinación en la elaboración de proyectos curriculares de centro; intervención en programas de apoyo al curriculum; etc.

2. En el entorno de la orientación profesional: orientación laboral a colectivos con acceso restringido (mujeres, mayores de cuarenta años, colectivos excluidos socialmente); información, gestión, acompañamiento y seguimiento de las inserciones laborales de inmigrantes; formación en estrategias de búsqueda de empleo; orientación y ayuda al alumnado universitario de final de carrera y en el tránsito hacia el mercado laboral; etc.

3. Menores en riesgo de desatención y descuidado familiar; información y formación familiar y social; acciones preventivas de carácter comunitario destinados a la infancia y juventud; atención educativa personalizada a menores bajo tutela administrativa; atención educativa personalizada a menores con medidas judiciales; etc.

4. En el ámbito de la atención a la diversidad y la educación inclusiva. Apoyo educativo fuera del sistema educativo a personas con necesidades especiales y sus familias; estimulación temprana e intervención en trastornos del desarrollo; eliminación de barreras sociales; atención educativa a personas dependientes y sus familias; servicios de psicomotricidad; intervención con jóvenes y adolescentes con comportamientos disruptivos y conflicto social; etc.

5. Intervención comunitaria, en general. Asesoramiento e intervención psicopedagógica en la edad adulta o formación a lo largo de la vida; atención a mayores, a través de programas y acciones de estimulación física y cognitiva; intervención hospitalaria; programas de intervención comunitaria en todos los ámbitos de acción psicopedagógica; etc. 
La incorporación de estos nuevos ámbitos al ejercicio profesional de la psicopedagogía está suponiendo un constante reto, tanto para investigadores como para profesionales que llevan a cabo la acción psicopedagógica. Además evidencia el arraigo y demanda social de los profesionales de la psicopedagogía. En algunas comunidades autónomas se ha invertido en el estudio de las competencias profesionales del psicopedagogo, cara al diseño de los nuevos planes de estudio de grado y postgrado. Este es el caso de la Comunidad Autónoma Andaluza, que financia, a través de la Consejería de Innovación, Ciencia y Empresa de la Junta de Andalucía, el proyecto denominado "Elaboración de guías docentes de titulaciones andaluzas conforme al sistema de créditos europeos (ECTS): las competencias de acción de psicopedagogía" (Angulo, Corpas, García, González, 2006). En este proyecto colaboran las Facultades de Educación de Cádiz, Ceuta, Córdoba, Granada, Huelva, Jaén, Málaga y Melilla y, participan, en la identificación de competencias, el profesorado que imparte docencia en la titulación de psicopedagogía, el alumnado de dicha titulación, y los profesionales de las provincias indicadas.

\section{Conclusiones}

1. Tras 15 años de institucionalización de la psicopedagogía en el Estado Español, y la Comunidad Autónoma Canaria, se puede afirmar que se ha consolidado un espacio disciplinar, en el que la investigación, la especialización y la ampliación de los ámbitos del ejercicio profesional se ven respaldadas bajo las nuevas demandas sociales.

2. A la psicopedagogía se le presenta un amplio horizonte en el siglo XXI. Abrir de nuevo el debate sobre el establecimiento de fronteras entre lo pedagógico y lo psicológico supone:

- Duplicar de nuevo funciones entre dos profesionales, que recibirán la misma formación, si bien con un carácter más pedagógico o psicológico dependiendo de donde proceda. Esta realidad supondría un desorden en la inserción profesional, ordenada actualmente en torno al profesional de la psicopedagogía.

- Duplicar la formación, tanto inicial como especializada, pues los grandes ámbitos de acción psicopedagógica se han desarrollado y se desarrollan en la actualidad indistintamente por las dos disciplinas.

- Fragmentar y perder las referencias profesionales en un espacio de convergencia europea de educación superior. Los profesionales de la educación, como tantos otros profesionales, se caracterizan por la formación interdisplinar. Por otro lado, en un contexto 
en el que se hacen esfuerzos por converger en perfiles profesionales generales a un espacio europeo, no tiene sentido la vuelta a la superespecialización. Tiene sentido, en todo caso, como dice la normativa de Grados y Postgrados, un postgrado especializado, no profesionalizador, y menos cuando sus funciones coinciden con otras titulaciones de grado en vigor.

3. El amplio espacio profesional reclama una formación inicial de grado generalista, que dé paso a postgrados especializados en ámbitos específicos tales como: orientación laboral; atención a la diversidad; menores bajo tutela administrativa (abandono familiar, medidas judiciales, inmigrantes no acompañados); etc.

4. En un mundo globalizado y en una Europa que pretende un espacio común de educación, y ante unas necesidades sociales que cada día reclaman más de la interdisplinariedad, la psicopedagogía se erige como modelo de convergencia epistemológica de muchas disciplinas y de muchos ámbitos de acción profesional. La idea de interdisciplinariedad conlleva unos planteamientos que sean integradores de las diferentes perspectivas profesionales frente a la dicotomía que se establece en la división multidisciplinar. Se debe superar el enfrentamiento gremial que rivaliza y compite por un espacio profesional que ha de ser común, desterrando la perspectiva de un pensamiento patrimonialista que cierra las posibilidades a los futuros profesionales de la psicopedagogía y reduce los beneficios a las personas destinatarias de los servicios psicopedagógicos.

5. En suma, desde la puesta en marcha de la Licenciatura de Psicopedagogía en el Estado Español y ante la perspectiva de la reforma europea de la enseñanza universitaria, con el surgimiento de los nuevos títulos de Grado y de Postgrado, se hace necesario continuar avanzando en la consolidación de un espacio científico y profesional común, en el cual convivan pacíficamente los profesionales de la Psicología y de la Pedagogía, en aras de seguir profundizando en el diseño, implementación y optimización de los modelos de prestación de servicios psicopedagógicos, que redunden en el bienestar de las personas beneficiarias de los mismos y en la mejora de la calidad educativa. Asimismo, a la psicopedagogía se le van a ir abriendo nuevos contextos y escenarios de actuación, tanto científica como profesional, que habrán de requerir del esfuerzo conjunto de las ciencias psicológicas y pedagógicas. 


\section{Referencias}

Álvarez, P., Bethencourt, J. y Cabrera, L. (2000). La transición al mercado laboral de los psicopedagogos: estudio de las dos primeras promociones en la Universidad de la Laguna. Revista de Psicología General y Aplicada, 53(3), 535-547.

Andrey, B y Le Men, J (1970). La psychologie a l'ecole. París: Presses Universitaires de France. Edición castellana: Madrid, Studium.

Angulo, J., Corpas, M. C., García, J. D., y González, I. (2006). Las competencias de la titulación de psicopedagogía a nivel andaluz: Investigando la opinión del profesorado, del alumnado universitario y de los profesionales de la orientación. Revista de Investigación Educativa, 24(2), 575-594.

Báez, B. y Bethencourt, J. T. (1992). Psicología escolar. Buenos Aires: Cincel.

Bisquerra, R. (1996). Orígenes y desarrollo de la orientación psicopedagógica. Madrid: Narcea.

Cano, J. (2003). Diversidad de roles del orientador psicopedagógico: Posibilidades y dificultades en su trabajo profesional. Anuario de Pedagogía, 5, 103-119.

Carrión, I. (1996). Orígenes de la psicopedagogía. Campo Abierto: Revista de Educación, 18, 229-248.

Castro, J. R., Rodríguez, I., y Cubo, S. (2001). El prácticum de psicopedagogía en la universidad de Extremadura: 1995-2001, en Zabalza, M., Iglesias, M., Raposo, M. y Cid, A. (Coords.). El practicum y las prácticas en empresas en la formación universitaria: desarrollo de competencias personales y profesionales. Actas del Simposium Internacional sobre Practicums.

Cerezo, M. A. (1996). Los comienzos de la psicopedagogía en la España contemporánea (1882-1936). Valladolid: Secretariado de Publicaciones e Intercambio Científico; Universidad de Valladolid.

Cerezo, M. A. (2001). Los comienzos de la psicopedagogía en España (1882-1936). Madrid: Biblioteca Nueva.

Coll, C. (1996). Psicopedagogía: confluencia disciplinar y espacio profesional. En Monereo, C. y Solé, I. (Coords.) El asesoramiento psicopedagógico: una perspectiva profesional y constructivista. Madrid: Alianza.

Cubo, S. y Montenero, M. (2000). El practicum de psicopedagogía de la facultad de Educación de la Universidad de Extremadura. Campo Abierto: Revista de Educación, 18, 133-162. 
Gonzalo, M.L. (1991). La profesionalización de las actividades de orientación e intervención psicopedagógica en el contexto de la reforma del sistema educativo. Bordón: Revista de Pedagogía, 43(2), 197-204.

Hidalgo, V. y Hidalgo, E. (2003). Breve reflexión: practicum de psicopedagogía, propulsor del futuro profesional del psicopedagogo/a. En Coriat Benarroch, Romero López y Gutiérrez Pérez. El practicum en la formación inicial del profesorado de magisterio y educación secundaria: avances de investigación, fundamentos y programas de formación. Granada: Editorial Universidad de Granada.

López, R. (2001). El practicum de psicopedagogía en la universidad: reflexión y análisis descriptivo desde una experiencia de intervención. En Zabalza Beraza, M.A., Iglesias Forneiro, M.L., Raposo Rivas, M. y Cid Sabucedo, A. El practicum y las prácticas en empresas en la formación universitaria: desarrollo de competencias personales y profesionales en el practicum.

MEC (1990). Orientación educativa y la intervención psicopedagógica. Madrid: MEC

Moreu, A. C. (2002). La fundamentación contemporánea del discurso psicopedagógico. Historia de la Educación: Revista Interuniversitaria, 21, 105-116.

Moreu, A. C. y Bisquerra Alzina, R. (2002). Los orígenes de la psicopedagogía: El concepto y el término. Revista Española de Orientación y Psicopedagogía, 13(1), 17-29.

Oakland, T. y Cunnigham, J. L. (2003). Definition of school psychology. En http://www.ispaweb.org/Documents/definition_fulldoc.html

Ocampo, C. y Cid , A. (2001). Funciones tutoriales en el practicum de magisterio y psicopedagogía, en Zabalza, M., Iglesias, M., Raposo, M. y Cid, A. (Coords.). El practicum y las prácticas en empresas en la formación universitaria: desarrollo de competencias personales y profesionales. Actas del Simposium Internacional sobre Practicums.

Ortiz, E. (2000). Una comprensió epistemològica de la psicopedagogia. Temps d'Educacio, 24, 191-202.

Pérez, M. P. y Torres, J. (1998). El prácticum de pedagogía y psicopedagogía en la universidad pontificia de comillas de Madrid: Descripción y singularidades. Diputación de Pontevedra.

Poveda, D. (2003). La psicopedagogía como disciplina: Una perspectiva contextualista y de ciclo vital. Revista Española de Orientación y Psicopedagogía, 14(2), 105-119.

Rigo, E. (1990). La psicopedagogía: Un nuevo perfil profesional o de cómo buscar la coherencia, en La reforma de los estudios universitarios en educación. La Laguna: Servicio de Publicaciones de la Universidad de La Laguna. 
Tello Díaz, J. (2007). El practicum de la licenciatura de psicopedagogía de la universidad de Huelva: implicaciones en la formación del psicopedagogo y su incidencia en la apertura de yacimientos de empleo. Tesis Doctoral. Universidad de Huelva. 